\title{
The Universe as a Resonant Cavity: a Small Step Towards Unification of MoND and MiHsC
}

\author{
Keith A. Pickering ${ }^{1}$ \\ 10085 County Road 24, Watertown, MN 55388 United States \\ Email: keith.pickering@gmail.com
}

\begin{abstract}
The physics of hot, dense plasma implies that there was a time shortly after the Big Bang when the universe was a perfectly conductive and opaque medium. Therefore, looking deep into space and time, our universe can be considered to be surrounded by a perfectly conductive shell of that plasma, and is therefore a resonant cavity. This insight allows computation of a new value for $\Theta$, the longest allowable wavelength in the universe, which is somewhat higher than previously accepted. The new value of $\Theta=3.4125 H_{0}$ resolves about half of the difference between the predictions of MoND and MiHsC for the minimum possible acceleration in the universe, and about $35 \%$ of the difference in galactic rotational velocity.
\end{abstract}

Keywords: MoND, MiHsC, resonant cavities

\section{Introduction}

In the broadest sense, a resonant cavity is a region of space bounded by a conductor that is opaque and reflective of photons. The physics of resonant cavities are important in radio and microwave communication, and have been investigated extensively starting with Rayleigh in 1897. Photons inside a cavity resonate that is, they form standing waveforms - in several modes. These modes are classified as TE, TM, and TEM, depending on whether the waveforms are transverse to the electrical plane, transverse to the magnetic plane, or transverse to both electrical and magnetic planes. Modes are further categorized according to the number of waveforms that resonate inside the cavity across three dimensions of space, which can be defined in any coordinate system. In microwave physics these three dimensions are represented as $a, b$, and $d$, while their associated mode numbers are subscripted $m, n$, and $p$.

As we look deeper and deeper into space, we look farther and farther back in time, eventually reaching the time shortly after the Big Bang when a hot, dense plasma of electrons and protons condensed to form neutral atoms. At that point the Universe became transparent and the photons then present in the Universe were released to form the Cosmic Microwave Background (CMB). In the physics of dense hydrogen plasma - and here we use "hydrogen" to mean a plasma composed of equal numbers of electrons and protons - it has been shown [1] that as the density of the plasma increases, its relative permittivity $\varepsilon$ increases from 1 (the relative permittivity of free space) towards infinity at a critical value of $q$, where $q$ is a dimensionless wavenumber combining inverse plasma density with energy density.

As $\varepsilon$ approaches infinity, the conductivity of the plasma also approaches infinity, and the plasma becomes perfectly conductive and opaque to photons of all wavelengths. Therefore as we look deep into space towards that point in time, the Universe can be considered as being surrounded by a shell of perfectly conductive metallic hydrogen plasma. And therefore, the Universe as a whole can be considered to be a spherical resonant cavity with a radius of $H_{0}$, the Hubble length. The blackbody radiation from this shell, greatly redshifted, can be observed as the CMB.

\section{Resonant Cavities}

Spherical resonant cavities have not been extensively investigated because their usefulness in communication is limited. However, we can consider as a close analog a cylindrical resonant cavity with an identical radius $H_{0}$ and a length of $2 H_{0}$, where a photon of interest is at the center of the cylinder moving along the axis. Cylindrical cavities are useful in communications as waveguides, and their properties are well 
known. For a cavity of given geometry and a given resonant mode, there is a cutoff frequency $f_{c}$, below which waves will not resonate in the cavity. Every resonant mode has a different cutoff frequency, but for a given cavity there is one resonant mode with the lowest cutoff frequency, called the dominant mode of the cavity. For cylindrical wavguides, $\mathrm{TE}_{11}$ is the dominant mode with the lowest cutoff frequency. (Note that in general waveguides have an indeterminate length $d$, so the modenumber $p$ is not defined.) The cutoff wavelength of the dominant mode therefore defines waves that are too large to fit inside the cavity in any mode.

Following [2], the cutoff frequency for mode $m n$ of a cylindrical waveguide is given by

$$
\left(f_{c}\right)_{m n}=\frac{\chi_{m n}^{\prime}}{2 \pi a \sqrt{\mu \varepsilon}}
$$

where dimension $a=b$ is the radius of the cavity, $\chi_{m n}^{\prime}$ is the zero solution of the first derivative $J_{m}^{\prime}\left(\chi_{m n}\right)$ of Bessel function $J_{m}(x)$; and $\mu$ and $\varepsilon$ are the permeability and permittivity of the medium inside the cavity. For a vacuum, $\varepsilon_{0} \equiv 1 /\left(\mu_{0} c^{2}\right)$, hence $\sqrt{\mu_{0} \varepsilon_{0}}=1 / c$. Then setting $a=b=H_{0}$, and using dominant mode $\mathrm{TE}_{11}$, we derive a value for $\Theta$, the longest wavelength allowed in the Universe:

$$
\Theta=\frac{2 \pi H_{0}}{\chi_{1,1}^{\prime}}
$$

For $\mathrm{TE}_{11}$ the Bessel derivative zeroes at $\chi_{1,1}^{\prime}=1.8412$ [2], and with a Hubble length $H_{0}=1.27 \times 10^{26}$ $\mathrm{m}$ [3], the longest possible wavelength is

$$
\Theta=3.4125 H_{0} \approx 4.3 \times 10^{26} \mathrm{~m}
$$

This result is greater by a factor of $\pi / \chi_{1,1}=1.71$ from the maximum wavelength proposed in [4], defined there as $\Theta=2 H_{0}$. The limit is absolute, and therefore sets a limit on blackbody radiation and Unruh radiation as well.

\section{Discussion}

While both MoND [5] and MiHsC [4] provide new physics that explains galaxy rotation curves without resorting to Dark Matter, MoND has one free parameter, $a_{0}$, that is determined empirically, while MiHsC has no free parameters, deriving its results only from universal constants. Therefore a change to one of those underlying constants used by MiHsC in a direction that brings the two theories somewhat closer can be seen as a modest advance.

The parameter $a_{0}$ is the minimum acceleration allowed in MoND, determined in [6] to be $a_{0}=$ $1.24 \pm 0.14 \times 10^{-10} \mathrm{~ms}^{-2}$, derived from a fit to low surface brightness galaxies. The minimum acceleration allowed in $\mathrm{MiHsC}$ is $2 c^{2} / \Theta$. The difference between the theories is summarized below.

Table 1. Comparison of difference between MoND, MiHsC in its usual form, and the present work.

\begin{tabular}{llll}
\hline Quantity, units & MoND & MiHsC, ref. [4] & MiHsC, present work \\
\hline Minimum acceleration, $\times 10^{-10} \mathrm{~m} \mathrm{~s}^{-2}$ & 1.24 & 7.13 & 4.18 \\
Minimum rotational velocity for galaxy of $10^{11} M \odot, \mathrm{km} \mathrm{s}^{-2}$ & 358 & 555 & 485 \\
\hline
\end{tabular}

Thus we have eliminated about $35 \%$ of the discrepancy between MoND and MiHsC in rotational velocity for typical galaxies, and about half the discrepancy in acceleration. 


\section{References}

1. J. P. Hansen and I. R. McDonald, "Microscopic simulation of a strongly coupled hydrogen plasma," Phys. Rev. A, vol. 23, pp. 2041-2059, Apr 1981. [Online]. Available: http://link.aps.org/doi/10.1103/PhysRevA.23.2041

2. C. A. Balanis, Advanced engineering electromagnetics. John Wiley \& Sons, 2012.

3. A. G. Riess, L. M. Macri, S. L. Hoffmann, D. Scolnic, S. Casertano, A. V. Filippenko, B. E. Tucker, M. J. Reid, D. O. Jones, J. M. Silverman et al., "A $2.4 \%$ determination of the local value of the hubble constant based on observations with the nasa/esa hubble space telescope, obtained at the space telescope science institute, which is operated by aura, inc., under nasa contract nas 5-26555." The Astrophysical Journal, vol. 826, no. 1, p. 56, 2016.

4. M. McCulloch, "Modelling the pioneer anomaly as modified inertia," Monthly Notices of the Royal Astronomical Society, vol. 376, no. 1, pp. 338-342, 2007.

5. M. Milgrom, "A modification of the newtonian dynamics as a possible alternative to the hidden mass hypothesis," The Astrophysical Journal, vol. 270, pp. 365-370, 1983.

6. S. S. McGaugh, "Novel test of modified newtonian dynamics with gas rich galaxies," Phys. Rev. Lett., vol. 106, p. 121303, Mar 2011. [Online]. Available: http://link.aps.org/doi/10.1103/PhysRevLett.106.121303 